\title{
LED Lighting System Reliability Modeling and Inference via Random Effects Gamma Process and Copula Function
}

\author{
Huibing Hao, ${ }^{1,2}$ Chun Su, ${ }^{1}$ and Chunping $\mathrm{Li}^{2}$ \\ ${ }^{1}$ Department of Industrial Engineering, Southeast University, Nanjing 211189, China \\ ${ }^{2}$ Department of Mathematics, Hubei Engineering University, Xiaogan 432100, China \\ Correspondence should be addressed to Chun Su; haohuibing1979@163.com
}

Received 13 December 2014; Accepted 15 January 2015

Academic Editor: Ahmad Umar

Copyright (c) 2015 Huibing Hao et al. This is an open access article distributed under the Creative Commons Attribution License, which permits unrestricted use, distribution, and reproduction in any medium, provided the original work is properly cited.

\begin{abstract}
Light emitting diode (LED) lamp has attracted increasing interest in the field of lighting systems due to its low energy and long lifetime. For different functions (i.e., illumination and color), it may have two or more performance characteristics. When the multiple performance characteristics are dependent, it creates a challenging problem to accurately analyze the system reliability. In this paper, we assume that the system has two performance characteristics, and each performance characteristic is governed by a random effects Gamma process where the random effects can capture the unit to unit differences. The dependency of performance characteristics is described by a Frank copula function. Via the copula function, the reliability assessment model is proposed. Considering the model is so complicated and analytically intractable, the Markov chain Monte Carlo (MCMC) method is used to estimate the unknown parameters. A numerical example about actual LED lamps data is given to demonstrate the usefulness and validity of the proposed model and method.
\end{abstract}

\section{Introduction}

In recent years, significant advances have been achieved in the manufacturing of light emitting diode (LED). At present, LED manufacturers have successfully designed LED as an indicator for many devices in the lighting systems due to the benefit of its low energy and long lifetime [1]. For its longer lifetime, higher reliability, and different failure mechanisms, it is difficult to evaluate and predict the reliability of LED. In order to study this, an understanding of the possible failure mechanisms of LED lamp should be examined.

In general, the failure mechanisms of a system can be categorized into catastrophic failures and degradation failures [2]. Catastrophic failures are characterized as being both complete and sudden. A failure is considered as a catastrophic failure which means the system ceases to operate. Degradation failures are characterized as being both partial and gradual. If a system is considered to have a degradation failure, the system still functions but does not meet the performance target. It is well known that the light output form power LED is highest when new and declines gradually over time; hence, that is a degradation failure. From the result in [3], we know that the common failure modes of LED can be divided into three failure modes, including catastrophic failure, intensity degradation failure, and chromatic change degradation failure. Because the probability of catastrophic failure under standard aging test conditions is very low [4], then, for the LED, the intensity degradation and chromatic change degradation are the domination failure modes.

Degradation (e.g., wear, erosion, and fatigue) is a common phenomenon for most mechanical systems or components. Degradation can be mathematically described with a continuous process in terms of time [5]. Because the stochastic process model can provide flexibility to describe the failure generating mechanisms and the operating environment characteristics, many articles use the stochastic process approach to model the degradation path, such as Markov chain, Wiener processes, and Gamma processes [68].

Most of the previous research which focuses on degradation analysis only considers one performance characteristic (PC) or component failure mechanism. In practice, modern 
products usually have complex structure and more functions. This means that modern highly reliable products may have multiple degradation failure mechanisms. A motivating example is a lighting system consisting of many light emitting diode (LED) lamps for different purposes of lighting. LED lamp has attracted increasing interest in the field of lighting systems due to its low energy and long lifetime. For different functions (i.e., illumination and color), it may have two or more PCs. In such situations, multivariate or at least bivariate degradation model is needed to estimate the reliability of products.

There are some works dealing with estimating system reliability of bivariate or multivariate degradation data [914]. These works use either independence assumption of the multiple PCs, multivariate normal distribution, or modeling with covariates and consider modification to single failure classifications. However, these assumptions may not match the engineering practice. In $[15,16]$, Sari et al. answered the question as to how one could quantify the reliability of LED lighting system which had two or more degraded PCs, and the dependence of these PCs was described through a copula function. But in Sari's work, they modeled the degradation data with generalized linear regression model with population average approach. Compared with stochastic process model, the regression model ignores the temporal uncertainty of a degradation process, which results in limiting its applications. Hence, based on Sari's work, Pan et al. [17, 18] used the Wiener process and the copula function to model multiple degradation failure mechanism, and a numerical example about fatigue cracks was presented.

From the definition of Wiener process, it is known that the degradation path is not a strictly increasing function. Generally speaking, a gamma process is better suited for modeling a degradation behavior that has a strictly increasing pattern. Crowder and Lawless [19] used a gamma process to illustrate their single-inspection policy for the maintenance of automobile brake pads. van Noortwijk [20] recently surveyed the application of gamma processes in maintenance. Tsai et al. [21] discussed the misspecification effect on the prediction of product's MTTF when the degradation model is fitted by gamma and Wiener degradation processes. Bagdonavicius and Nikulin [22] discussed a joint model including gamma degradation process and catastrophic failures. Wang et al. [23] used bivariate gamma degradation process to model the degradation data and an adaptive method of residual life was obtained.

In addition, similar to Sari's work, Pan et al. [17, 18] also only use the population average approach and do not consider the random effect in their model. In fact, considering that each product possibly experiences different sources of variations during its operation, for a degradation model to be realistic, it is more appropriate to incorporate unit to unit variability in the degradation process. The degradations of such products can be described by random effects models, where the random effects can capture the unit to unit differences. Particularly for the actual LED lighting system, there are few researches using the copula method with the random effects Gamma process to assess the products reliability.
From the above analysis, it is clear that the reliability assessment of the system with two PCs has not been studied thoroughly. In this paper, we assume that the LED lighting system has two PCs (i.e., illumination and color), and each PC is governed by a random effects Gamma process. Moreover, we assume that the two PCs are dependent and their dependency can be characterized by a copula function. Since the likelihood function in such a situation is quite complicated, the Markov chain Monte Carlo (MCMC) algorithm is used to obtain the unknown parameters.

The rest of the paper is organized as follows. In Section 2, some assumptions and copula basic are described. Then, the bivariate degradation model based on random effects Gamma process and the copula function is introduced in Section 3. In Section 4, the estimation of unknown parameters based on the MCMC algorithm is obtained. A numerical example about actual LED lamps data is given in Section 5. Finally, some conclusions are made in Section 6.

\section{Some Basic Assumptions and Copula Brief Introduction}

2.1. Basic Assumptions. To analyze the problem statistically, some assumptions are used for the reliability modeling in this paper. The details of each assumption are explained in the corresponding sections.

(1) The samples are independent, and no catastrophic failures occur during the degradation process.

(2) The marginal degradation processes can be modeled as Gamma process with random effect.

(3) For a specific product, the degradation measurements on the two PCs are observed at the same time (balanced data).

(4) A product is supposed to have failed if one of the PCs exceeds the corresponding failure threshold for the first time. Here, the failure threshold vector is denoted by $\xi=\left(\xi_{1}, \xi_{2}\right)$.

(5) The two PCs are dependent on each other, and the dependency can be characterized by a Frank copula function.

\subsection{Copulas and Their Properties}

2.2.1. Definition and Base Properties. Copula function is a powerful tool to model the dependence structure among multiple PCs since they (see Nelsen [24]) give the dependence structure which relates the known marginal distributions of each PC to their multivariate joint distribution.

A two-dimensional copula $C(u, v)(u \in[0,1], v \in$ $[0,1])$ is conventionally defined as a bivariate cumulative distribution function with uniform margins. A probabilistic way to define the copula is provided by the theorem of Sklar (see Nelsen [24]).

Theorem 1. Let $X$ and $Y$ be random variables with continuous distributions $F(x)$ and $G(y)$, respectively, and let $H(x, y)$ be the 
two-dimensional cumulative distribution function. Then, there exists a two-dimensional copula $C(\cdot, \cdot)$ such that, for all $x, y \in$ $(-\infty,+\infty)$

$$
H(x, y)=C(F(x), G(y)) .
$$

If $F(\cdot)$ and $G(\cdot)$ are continuous, then $C(\cdot, \cdot)$ is unique; conversely, if $C(\cdot, \cdot)$ is a copula and $F(\cdot)$ and $G(\cdot)$ are distribution functions, then the function $H(\cdot, \cdot)$ defined by (1) is a joint distribution function with margins $F(\cdot)$ and $G(\cdot)$.

2.2.2. Measures of Association. We will consider here the standard dependence measures, Spearman's $\kappa$ and Kendall's $\tau$. These measures are related to the copula since the latter is an expression of the stochastic relationship between $u$ and $v$ within the entire range of values the variables can take. It is not difficult to show that

$$
\begin{aligned}
& \kappa=12 \iint_{[0,1]^{2}} C(u, v) d u d v-3, \\
& \tau=3 \iint_{[0,1]^{2}} C(u, v) d C(u, v)-1,
\end{aligned}
$$

where the range of $\kappa$ and $\tau$ can be shown to be $[-1,1]$. For further properties of $\kappa$ and $\tau$, see Nelsen [24].

2.2.3. Archimedean Copula. Archimedean copulas have a wide range of applications, because

(a) they can be constructed easily;

(b) they have many nice properties;

(c) a lot of copulas belong to this class;

(d) they can be easily extended from 2-dimension to $m$ dimension when satisfying some conditions.

In this paper, the Frank copula which belongs to Archimedean copula family is used to depict the dependence among multiple PCs as follows:

$$
\begin{aligned}
& C(u, v) \\
& \quad=-\frac{1}{\theta} \ln \left\{1+\frac{[\exp (-\theta u)-1][\exp (-\theta v)-1]}{\exp (-\theta)-1}\right\},
\end{aligned}
$$

where $\theta$ is the Frank copula parameter and $\theta \in(-\infty, 0) \cup$ $(0,+\infty)$. And the relationship between Kendall's $\tau$ and the Frank copula parameter $\theta$ is given by

$$
\tau=1+4 \frac{D_{1}(\theta)-1}{\theta}
$$

where $D_{1}(\theta)=(1 / \theta) \int_{0}^{\theta}\left(t /\left(e^{t}-1\right)\right) d t$ is a Debye function of the first kind (see Nelsen [24]).

\section{Reliability Model Based on Gamma Process with Random Effects}

3.1. Marginal Reliability Model Based on Random Effects Gamma Process. As mentioned earlier, a gamma process is more suitable for describing a monotone increasing degradation path. A well-adopted form for the regular Gamma process $\{X(t), t \geq 0\}$ can be expressed as

$$
X(t) \sim \operatorname{Gamma}(\alpha t, \beta),
$$

where $\operatorname{Gamma}(\alpha t, \beta)$ is a Gamma distribution with shape parameter $\alpha t(\alpha>0)$ and scale parameter $\beta(\beta>0)$, and the corresponding probability density function (PDF) is

$$
f_{G}(x ; \alpha t, \beta)=\frac{\beta^{\alpha t}}{\Gamma(\alpha t)} x^{\alpha t-1} \exp [-\beta x], \quad x>0,
$$

where $\Gamma(s)=\int_{0}^{+\infty} x^{s-1} \exp (-x) d x$ is Gamma function, and the Gamma process has the following properties:

(1) $X(0)=0$ with probability one;

(2) $X(t)$ has independent increments; that is, $X\left(t_{2}\right)-$ $X\left(t_{1}\right)$ and $X\left(s_{2}\right)-X\left(s_{1}\right)$ are independent, $\forall t_{2}>t_{1}>$ $s_{2}>s_{1}$

(3) $\Delta X(t)=X(t+\Delta t)-X(t) \sim \operatorname{Gamma}(\alpha \Delta t, \beta), \forall \Delta t>0$.

Assume that the degradation path of a product is governed by (5). Given the threshold value $\xi>0$, the product's lifetime $T$ is defined as

$$
T=\inf \{t \mid X(t) \geq \xi\} .
$$

Since the Gamma process has a monotone path, then the cumulative distribution function (CDF) of the lifetime $T$ can be expressed as

$$
\begin{aligned}
F(t) & =P(T \leq t)=P(X(t) \geq \xi) \\
& =1-\frac{\beta^{\alpha t}}{\Gamma(\alpha t)} \int_{0}^{\xi} x^{\alpha t-1} \exp [-\beta x] d x .
\end{aligned}
$$

From the properties of the Gamma process, we know that the average performance degradation characteristic in (5) is a linear function about $t$. When it is not linear, Whitmore and Schenkelberg [25] used a transformed time scale $\Lambda(t)$ to make it linear. Generally, the transformation is denoted by

$$
\begin{aligned}
& \Lambda(t)=\Lambda(t, b)=t^{b}, \quad \text { or } \\
& \Lambda(t)=\Lambda(t, b)=1-\exp (b t) .
\end{aligned}
$$

Then, a nonstationary Gamma process can be written as

$$
X(t) \sim \operatorname{Gamma}(\alpha \Lambda(t), \beta) .
$$

Considering that each item possibly experiences different sources of variations during its operation, for a degradation model to be realistic, it is more appropriate to incorporate item to item variability in the degradation process. In this paper, for simplicity, we assume that $b$ and $\alpha$ are the fixed parameters which are common to all products; $\beta$ is a random parameter representing the heterogeneity among different products. Then, the random effects Gamma process can be written as

$$
\begin{aligned}
X(t) & \sim \operatorname{Gamma}(\alpha \Lambda(t), \beta), \\
\beta & \sim \operatorname{Gamma}(\eta, \gamma),
\end{aligned}
$$


where $\operatorname{Gamma}(\eta, \gamma)$ is a Gamma distribution with shape parameter $\eta(\eta>0)$ and scale parameter $\gamma(\gamma>0)$, and the corresponding probability density function (PDF) of $\beta$ is

$$
g(\beta)=\frac{\gamma^{\eta}}{\Gamma(\eta)} \beta^{\eta-1} \exp [-\gamma \beta], \quad \beta>0 .
$$

Then, the PDF of $X(t)$ in (11) is given by

$$
\begin{aligned}
f_{X(t)}(x) & =\int_{0}^{+\infty} f_{G}(x ; \alpha \Lambda(t), \beta) g(\beta) d \beta \\
& =\frac{\Gamma(\alpha \Lambda(t)+\eta)}{\Gamma(\alpha \Lambda(t)) \Gamma(\eta)} \frac{\gamma^{\eta} x^{\alpha \Lambda(t)-1}}{(\gamma+x)^{\alpha \Lambda(t)+\eta}} .
\end{aligned}
$$

From (13), for any fixed $t$, the random variable $Y(t)=$ $\eta X(t) / \alpha \gamma \Lambda(t)$ has an $F$ distribution with $2 \alpha \Lambda(t)$ and $2 \eta$ degrees of freedom, and we denote $F_{2 \alpha \Lambda(t), 2 \eta}(y)$ as the CDF of $Y(t)$.

Then, the CDF of $X(t)$ in (11) is given by

$$
\begin{aligned}
F_{X(t)}(x) & =P(X(t) \leq x)=P\left(\frac{\eta X(t)}{\alpha \gamma \Lambda(t)} \leq \frac{\eta x}{\alpha \gamma \Lambda(t)}\right) \\
& =P\left(Y(t) \leq \frac{\eta x}{\alpha \gamma \Lambda(t)}\right) \\
& =F_{2 \alpha \Lambda(t), 2 \eta}\left(\frac{\eta x}{\alpha \gamma \Lambda(t)}\right) .
\end{aligned}
$$

Then, the CDF of the lifetime $T$ can be expressed as

$$
\begin{aligned}
F(t) & =P(T \leq t)=P(X(t) \geq \xi) \\
& =1-F_{2 \alpha \Lambda(t), 2 \eta}\left(\frac{\eta \xi}{\alpha \gamma \Lambda(t)}\right) .
\end{aligned}
$$

Suppose that a product has two PCs and each PC is governed by a random effects Gamma process. The $k$ th PC is defined by (11) as

$$
\begin{aligned}
X_{k}(t) & \sim \operatorname{Gamma}\left(\alpha_{k} \Lambda_{k}(t), \beta_{k}\right), \\
\beta_{k} & \sim \operatorname{Gamma}\left(\eta_{k}, \gamma_{k}\right),
\end{aligned}
$$

where $k=1,2$ and $\Lambda_{k}(t)=\Lambda\left(t, b_{k}\right)$.

Let $\xi_{k}$ be the threshold value of the $k$ th PC; the lifetime $T_{k}$ of the $k$ th PC is defined as

$$
T_{k}=\inf \left\{t \mid X_{k}(t) \geq \xi_{k}\right\} .
$$

Then, the failure time distribution of the $k$ th PC can be obtained as

$$
F_{k}(t)=1-F_{2 \alpha_{k} \Lambda_{k}(t), 2 \eta_{k}}\left(\frac{\eta_{k} \xi_{k}}{\alpha_{k} \gamma_{k} \Lambda_{k}(t)}\right)
$$

and the reliability function is

$$
R_{k}(t)=1-F_{k}(t)=F_{2 \alpha_{k} \Lambda_{k}(t), 2 \eta_{k}}\left(\frac{\eta_{k} \xi_{k}}{\alpha_{k} \gamma_{k} \Lambda_{k}(t)}\right) .
$$

3.2. Degradation Model Based on Bivariate Degradation Data. Suppose that a product has two PCs and the measurements of degradation process are random variables $X(t)=$ $\left(X_{1}(t), X_{2}(t)\right)$ at observation time point $t$. From the basic assumptions, the product is considered to be failed if any one PC reaches its corresponding failure threshold, which is known as $\xi=\left(\xi_{1}, \xi_{2}\right)$. Therefore, the product still works when each PC keeps below its failure thresholds. Given the failure time $T_{k}$ of the $k$ th PC, suppose that the lifetime of the system is $T$, and $T=\min \left(T_{1}, T_{2}\right)$. Then, the product reliability can be written as follows:

$$
\begin{aligned}
R(t) & =\operatorname{Pr}(T>t)=\operatorname{Pr}\left(T_{1}>t, T_{2}>t\right) \\
& =\operatorname{Pr}\left(X_{1}(t)<\xi_{1}, \quad X_{2}(t)<\xi_{2}\right) .
\end{aligned}
$$

If the two degradation failure mechanisms are assumed to be independent, the product reliability in (20) can be rewritten as

$$
\begin{aligned}
R(t) & =\operatorname{Pr}\left(X_{1}(t)<\xi_{1}, X_{2}(t)<\xi_{2}\right) \\
& =\operatorname{Pr}\left(X_{1}(t)<\xi_{1}\right) \times \operatorname{Pr}\left(X_{2}(t)<\xi_{2}\right) \\
& =R_{1}(t) \times R_{2}(t) .
\end{aligned}
$$

From (19) and (21), the product reliability with two PCs can be easily obtained. However, if the degradation failure mechanisms are not independent of each other, we cannot provide accurate product reliability estimation. Therefore, the copula method is utilized to establish the dependent structure among various degradation measurements.

Copulas provide a very convenient way to model and measure the dependence among multiple degradation failure mechanisms. The advantage of the copula function is that the joint reliability function can be modeled directly through the univariate marginal reliability functions of the individual failure processes, and the copula has no constraints on the univariate marginal distribution.

Suppose that $F_{k}(t)=1-R_{k}(t)$ is the CDF of lifetime $T_{k}$ for each PC, and $H\left(t_{1}, t_{2}\right)$ is the joint CDF of $T_{1}$ and $T_{2}$. According to Sklar's theorem, there exists a unique copula $C$ such that

$$
\begin{aligned}
P\left(T_{1} \leq t_{1}, T_{2} \leq t_{2}\right) & =H\left(t_{1}, t_{2}\right) \\
& =C\left(F_{1}\left(t_{1}\right), F_{2}\left(t_{2}\right) ; \theta\right),
\end{aligned}
$$

where $\theta$ is the parameter vector of the copula function.

Then, similarly to $[16,18]$, the system reliability in $(20)$ can be obtained as

$$
\begin{aligned}
R(t)= & P(T>t)=P\left(T_{1}>t, T_{2}>t\right) \\
= & 1-P\left(T_{1} \leq t\right)-P\left(T_{2} \leq t\right) \\
& +P\left(T_{1} \leq t, T_{2} \leq t\right) \\
= & R_{1}(t)+R_{2}(t)-1+C\left(F_{1}(t), F_{2}(t) ; \theta\right) .
\end{aligned}
$$


If the product has two PCs linked by bivariate Frank copula given in (3), then we can obtain the system reliability function as

$$
\begin{aligned}
& R(t) \\
& =R_{1}(t)+R_{2}(t)-1 \\
& \quad-\frac{1}{\theta} \ln \left\{1+\frac{\left[\exp \left(-\theta F_{1}(t)\right)-1\right]\left[\exp \left(-\theta F_{2}(t)\right)-1\right]}{\exp (-\theta)-1}\right\} .
\end{aligned}
$$

\section{Statistical Inferential Methods for Unknown Parameters}

Here, we discuss the estimation of parameters required to implement the reliability function in (24). The unknown parameters are

$$
\rho=\left(\alpha_{1}, \eta_{1}, \gamma_{1}, b_{1}, \alpha_{2}, \eta_{2}, \gamma_{2}, b_{2}, \theta\right) .
$$

From (18), (19), and (24), we know that the model not only has nine parameters, but also is very complicated from a computational viewpoint. For this reason, the MCMC with the Gibbs sampling techniques is employed in this study to estimate model parameters.

The MCMC method is a simulation technique in which the analytical posterior distribution is difficult to be computed. A Markov chain is generated by sampling the current point based on the previous one. The MCMC method works successfully in Bayesian computing. By using the MCMC method, it is possible to generate samples from the posterior distribution and to use these samples to estimate the desired features of the posterior distribution. In addition, the simulation algorithm can be easily extensible to models with a large number of parameters or high complexity. The MCMC techniques, including the Metropolis-Hastings (M$\mathrm{H})$ algorithm and the Gibbs sampler, have become very popular in recent years as methods for generating a sample from a complicated model (see the details in $[26,27]$ ).

The Gibbs sampler is a special case of an MCMC algorithm. It generates a sequence of samples from the full conditional probability distributions of two or more random variables. Gibbs sampling requires decomposing the joint posterior distributions into full conditional distributions for each parameter in the model and then sampling from them. We can use the Gibbs sampler to generate a sample, and then the unknown parameters are estimated. Let $\pi\left(\rho_{j} \mid \rho_{-j}, X\right)$ denote the full conditional posterior distribution of $\rho_{j}$, where $\rho_{-j}=\left(\rho_{1}, \ldots, \rho_{j-1}, \rho_{j+1}, \ldots, \rho_{n}\right)$ and $X$ is the observed data.

Then, the MCMC algorithm with the Gibbs sampling can be summarized as follows.

Step 1. Initialize $\rho^{(0)}=\left(\rho_{1}^{(0)}, \rho_{2}^{(0)}, \ldots, \rho_{n}^{(0)}\right)$.

Step 2. Set $t=1$.

Step 3. Generate $\rho_{1}^{(t)}$ from conditional distribution $\pi_{1}^{*}\left(\rho_{1} \mid\right.$ $\left.\rho_{2}^{(t-1)}, \rho_{3}^{(t-1)}, \ldots, \rho_{n}^{(t-1)}\right)$.

Step 4. Generate $\rho_{2}^{(t)}$ from conditional distribution $\pi_{2}^{*}\left(\rho_{2}\right.$ $\left.\rho_{1}^{(t-1)}, \rho_{3}^{(t-1)}, \ldots, \rho_{n}^{(t-1)}\right)$.
TABLE 1: Light intensity degradation data of 12 LEDs.

\begin{tabular}{ccccccc}
\hline \multirow{2}{*}{ Unit } & \multicolumn{5}{c}{ Inspection time (hour) } \\
& 0 & 50 & 100 & 150 & 200 & 250 \\
\hline PC1 & & & & & & \\
1 & 0 & 13.4 & 21.3 & 24.0 & 28.4 & 32.0 \\
2 & 0 & 17.9 & 28.6 & 34.6 & 38.3 & 42.0 \\
3 & 0 & 17.3 & 29.7 & 36.0 & 38.7 & 40.7 \\
4 & 0 & 20.2 & 31.7 & 37.7 & 40.0 & 41.0 \\
5 & 0 & 24.9 & 33.3 & 37.2 & 41.0 & 46.0 \\
6 & 0 & 16.3 & 26.0 & 32.6 & 37.0 & 38.7 \\
PC2 & & & & & & \\
1 & 0 & 27.0 & 35.0 & 39.3 & 41.7 & 42.0 \\
2 & 0 & 13.8 & 32.4 & 37.3 & 40.0 & 40.3 \\
3 & 0 & 18.8 & 35.0 & 39.4 & 40.7 & 42.7 \\
4 & 0 & 33.2 & 36.7 & 40.7 & 42.6 & 43.5 \\
5 & 0 & 33.9 & 35.8 & 40.6 & 42.0 & 44.7 \\
6 & 0 & 23.5 & 38.3 & 38.7 & 40.3 & 44.0 \\
\hline
\end{tabular}

Step 5. Generate $\rho_{j}^{(t)}$ from conditional distribution $\pi_{j}^{*}\left(\rho_{j} \mid\right.$ $\left.\rho_{1}^{(t-1)}, \ldots, \rho_{j-1}^{(t-1)}, \rho_{j+1}^{(t-1)}, \ldots, \rho_{n}^{(t-1)}\right)$.

Step 6. Generate $\rho_{n}^{(t)}$ from conditional distribution $\pi_{n}^{*}\left(\rho_{n} \mid\right.$ $\left.\rho_{1}^{(t-1)}, \rho_{2}^{(t-1)}, \ldots, \rho_{n-1}^{(t-1)}\right)$.

Step 7. Set $t=t+1$, and repeat steps $3-6, t=1,2, \ldots, N_{1}$.

Step 8 . Estimate desired features based on the simulate sample $\rho^{(m)}, \rho^{(m+1)}, \ldots, \rho^{\left(N_{1}\right)}$, where $m$ denotes the burnin number. For example, we can use the mean of $\rho_{j}^{(m)}, \rho_{j}^{(m+1)}, \ldots, \rho_{j}^{\left(N_{1}\right)}$ to be the estimator of $\rho_{j}$.

Using the Bayesian software package WinBUGS (see [28]) and carrying out the Gibbs sampling, the estimator of the model parameters can be obtained.

\section{Numerical Example: Application to a LED Lighting System}

In recent years, LED has attracted increasing interest in the field of lighting systems owing to its high efficiency, low energy, and long lifetime. Due to its different functions (i.e., illumination and color), there may have been multiple failure mechanisms about the LED lighting system. The actual LED lamps data are taken from Chaluvadi [29], where the LED lamps are put into test under an accelerated current of $40 \mathrm{~mA}$. In the original data, 12 samples are tested for LED data and the measurements are taken at the same measurement times. The measured frequency of its light intensity is 50 hours. For demonstrating the bivariate degradation model, similarly to Sari et al. [16] and Pan et al. [18], we choose 12 samples and the data will be treated as if half of it is the first PC and the other half represents the second PC. That is to say, in the LED lighting system, the performance demand of the first group LED lamps is for lighting, and the performance demand of the second group LED lamps is for chromatic change. The used data are the data measured only until 250 hours. The LED lighting system is considered to have failed if one of the two LED datasets is more than 50. Table 1 lists the LED data. 
TABle 2: Parameter estimation results considering the dependency.

\begin{tabular}{lcccc}
\hline Parameter & Mean & Standard error & MC error & 95\% HPD interval \\
\hline$\alpha_{1}$ & 3.783 & 1.304 & 0.03755 & $(1.657,6.715)$ \\
$\eta_{1}$ & 47.35 & 38.10 & 1.664 & $(6.00,150.20)$ \\
$\gamma_{1}$ & 42.29 & 34.62 & 1.503 & $(5.09,140.10)$ \\
$b_{1}$ & 0.4588 & 0.036 & $5.96 E-4$ & $(0.391,0.536)$ \\
$\alpha_{2}$ & 2.66 & 1.146 & 0.0247 & $(0.847,5.231)$ \\
$\eta_{2}$ & 28.38 & 24.57 & 1.009 & $(3.540,95.22)$ \\
$\gamma_{2}$ & 82.37 & 68.83 & 2.712 & $(10.59,268.0)$ \\
$b_{2}$ & 0.3226 & 0.052 & $8.84 E-4$ & $(0.235,0.442)$ \\
$\theta$ & 1.598 & 1.261 & $9.74 E-3$ & $(-0.87,4.112)$ \\
\hline
\end{tabular}

TABLE 3: Parameter estimation results without considering the dependency.

\begin{tabular}{lcccc}
\hline Parameter & Mean & Standard error & MC error & 95\% HPD interval \\
\hline$\alpha_{1}$ & 3.696 & 1.279 & 0.03584 & $(1.607,6.544)$ \\
$\eta_{1}$ & 48.97 & 39.14 & 1.363 & $(5.878,152.6)$ \\
$\gamma_{1}$ & 43.31 & 35.06 & 1.541 & $(4.931,139.7)$ \\
$b_{1}$ & 0.4592 & 0.037 & $5.61 E-4$ & $(0.390,0.537)$ \\
$\alpha_{2}$ & 2.613 & 1.112 & 0.0219 & $(0.868,5.176)$ \\
$\gamma_{2}$ & 28.83 & 24.28 & 0.9896 & $(3.486,97.74)$ \\
$\lambda_{2}$ & 81.68 & 68.29 & 2.681 & $(10.31,276.2)$ \\
$b_{2}$ & 0.3238 & 0.051 & $7.99 E-4$ & $(0.236,0.439)$ \\
\hline
\end{tabular}

5.1. Estimation of Unknown Parameters. Now, we use the LED data to illustrate the proposed model and method in this paper. van Noortwijk [20] and Tseng et al. [30] suggested that $\Lambda(t)=\Lambda(t, b)=t^{b}$ is appropriate for the LED degradation modeling, and this form is adopted in this paper. Considering the dependency between the two PCs, by using the MCMC method in Section 4, we generate 60,000 samples. A burn-in of 10,000 samples is used, with an additional 50,000 Gibbs samples used to estimate parameters. The prior distributions are given based on experience as

$$
\begin{aligned}
b_{k} & \sim \operatorname{Gamma}(0.001,0.001), \\
\alpha_{k} & \sim \operatorname{Gamma}(0.001,0.001), \\
\eta_{k} & \sim \operatorname{Gamma}(0.01,0.01), \\
\gamma_{k} & \sim \operatorname{Gamma}(0.01,0.01), \\
\theta & \sim N(0,0.001) .
\end{aligned}
$$

Table 2 tabulates posterior summaries including parameters posterior mean, standard error, Monte Carlo error, and 95\% HPD interval.

Meanwhile, the estimates of the unknown parameters without considering the dependency between the two PCs are presented in Table 3 .

5.2. Reliability Assessment. Based on the estimated results of the unknown parameters, the marginal reliability curves of $\mathrm{PC} 1$ and PC2 considering the dependency and independency are presented in Figures 1-2.

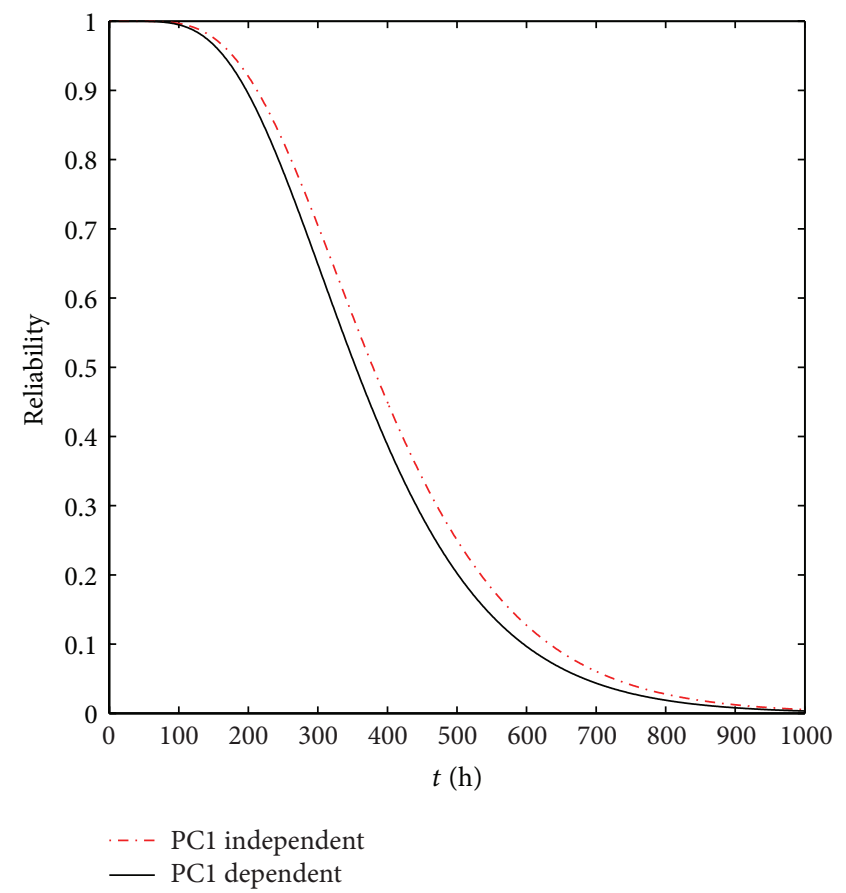

FIgURE 1: Marginal reliability curves of PC1.

It can be concluded from Figures 1 and 2 that either $\mathrm{PC} 1$ or PC2 has a relatively higher reliability under the case without considering the dependency than under the case considering the dependency. In other words, the incorrect independent assumption may overrate the reliability. 


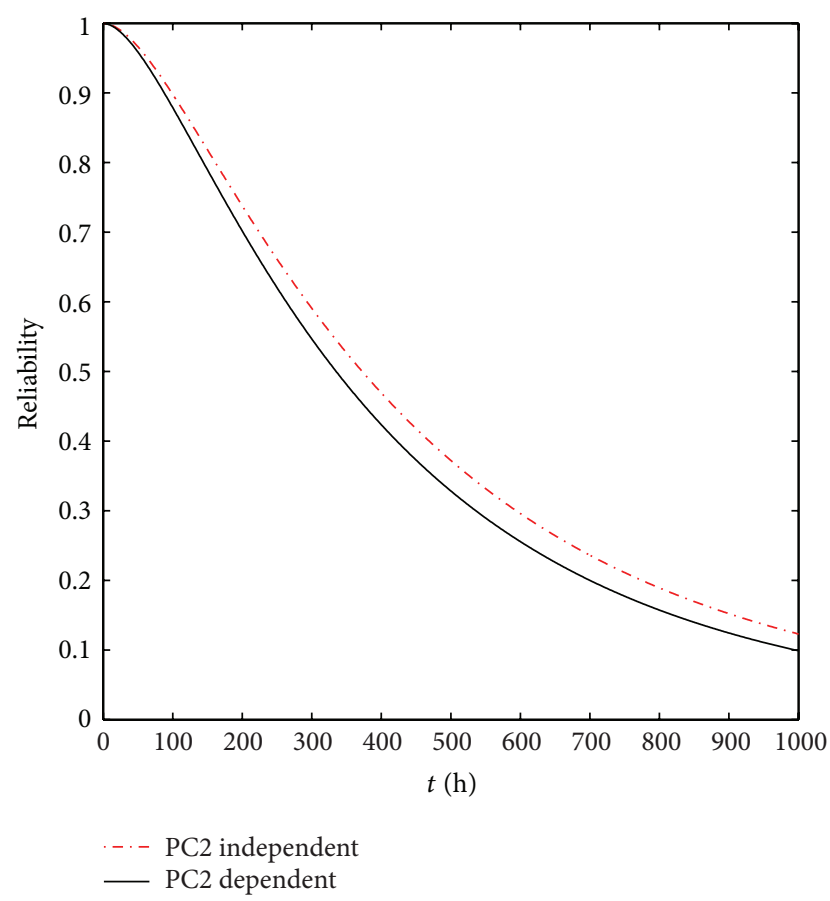

Figure 2: Marginal reliability curves of PC2.

Furthermore, the system reliability curves under the independent and dependent (with Frank copula assumption) cases are plotted in Figure 3. Comparing Figure 3 with Figures 1 and 2, we can find that there has been larger difference between the marginal reliability and the system reliability. Furthermore, from Figure 3, we can also find that there are some differences between the dependent and independent cases. That is to say, ignoring the dependence between PCs may result in different reliability conclusion. Therefore, it is necessary to analyze the possibility of the failure mechanisms dependency and perform the dependent reliability analysis.

\section{Conclusion}

In this paper, we establish a reliability model for the LED lighting system with two PCs, and each PC is governed by a random effects Gamma process. We suppose that the two PCs are dependent and the dependency is described by a copula function. The MCMC method is used to obtain the unknown parameters. From the numerical example of Section 5, we know that ignoring the dependence between PCs may result in different reliability conclusion.

In this work, we have only considered the case that products have two marginal distributions with a random Gamma process. It can be extended to the inverse Gaussian process, geometric Brownian motion, and so on. The different copula function (such as Gaussian copula and Gumbel copula) is also used to describe the relationship. Moreover, from the practice point of view, how to make effective maintenance decisions for the products with two PCs based on the proposed estimation results is necessary to be studied in the future.

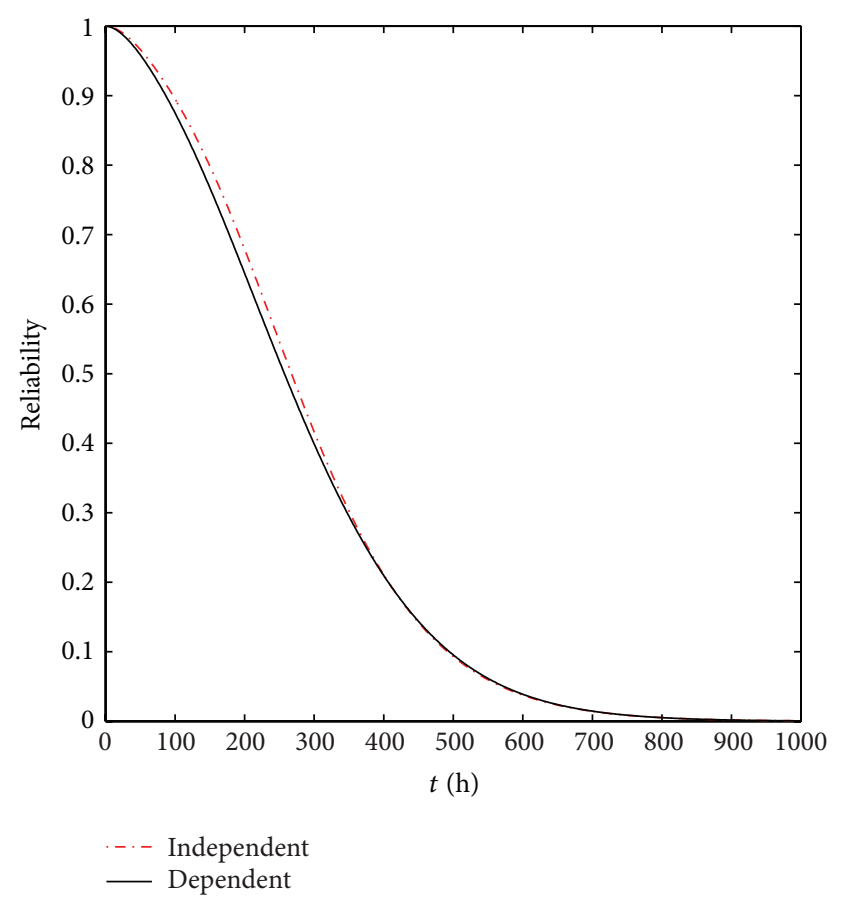

FIgURE 3: Reliability curves of system based on dependent and independent cases.

\section{Conflict of Interests}

The authors declare no conflict of interests regarding the publication of this paper.

\section{Acknowledgments}

The authors would like to thank the editor and the anonymous reviewers for their useful comments which have greatly improved the paper. This work is supported by the National Natural Science Foundation of China (no. 50405021), the Fundamental Research Funds for the Central Universities, the Graduate Training Innovative Projects Foundation of Jiangsu Province (no. CXLX12_0081), and the Youth Foundation of Hubei Educational Commission (no. Q20122603).

\section{References}

[1] E. F. Schubert and J. K. Kim, "Solid-state light sources getting smart," Science, vol. 308, no. 5726, pp. 1274-1278, 2005.

[2] W. Q. Meeker and L. A. Escobar, Statistical Method for Reliability Data, John Wiley \& Sons, New York, NY, USA, 1998.

[3] J. Fan, K. C. Yung, and M. Pecht, "Physics-of-failure-based prognostics and health management for high-power white light-emitting diode lighting," IEEE Transactions on Device and Materials Reliability, vol. 11, no. 3, pp. 407-416, 2011.

[4] L. Philps, "Evaluating the Lifetime Behavior of LED Systems," White paper, 2010.

[5] M. J. Zuo, R. Y. Jiang, and R. C. M. Yam, "Approaches for reliability modeling of continuous-state devices," IEEE Transactions on Reliability, vol. 48, no. 1, pp. 9-18, 1999. 
[6] S. J. Tang, C. Q. Yu, X. Wang, X. S. Guo, and X. S. Si, "Remaining useful life prediction of lithium-ion batteries based on the Wiener process with measurement error," Energies, vol. 7, no. 2, pp. 520-547, 2014.

[7] S.-T. Tseng, N. Balakrishnan, and C.-C. Tsai, "Optimal stepstress accelerated degradation test plan for gamma degradation processes," IEEE Transactions on Reliability, vol. 58, no. 4, pp. 611-618, 2009.

[8] J. P. Kharoufeh, D. E. Finkelstein, and D. G. Mixon, "Availability of periodically inspected systems with Markovian wear and shocks," Journal of Applied Probability, vol. 43, no. 2, pp. 303317, 2006.

[9] V. Crk, "Reliability assessment from degradation data," in Proceedings of the Annual Reliability and Maintainability Symposium-Product Quality and Integrity, pp. 155-161, RAMS, Los Angeles, Calif, USA, 2000.

[10] P. Wang and D. W. Coit, "Reliability prediction based on degradation modeling for systems with multiple degradation measures," in Proceedings of the Annual Reliability and Maintainability Symposium (RAMS '04), pp. 302-307, Los Angeles, Calif, USA, January 2004.

[11] D. Xu and W. B. Zhao, "Reliability prediction using multivariate degradation data," in Proceedings of the Annual Reliability and Maintainability Symposium on Product Quality and Integrity (RAMS '05), pp. 337-341, Alexandria, Va, USA, January 2005.

[12] W. Huang and R. G. Askin, "Reliability analysis of electronic devices with multiple competing failure modes involving performance aging degradation," Quality and Reliability Engineering International, vol. 19, no. 3, pp. 241-254, 2003.

[13] V. Bagdonavičius, A. Bikelis, and V. Kazakevičius, "Statistical analysis of linear degradation and failure time data with multiple failure modes," Lifetime Data Analysis, vol. 10, no. 1, pp. 65-81, 2004.

[14] V. Bagdonavicius, A. Bikelis, V. Kazakevicius, and M. Nikulin, "Analysis of joint multiple failure mode and linear degradation data with renewals," Journal of Statistical Planning and Inference, vol. 137, no. 7, pp. 2191-2207, 2007.

[15] J. K. Sari, Multivariate degradation modeling and its application to reliability testing [Ph.D. thesis], National University of Singapore, Singapore, 2007.

[16] J. K. Sari, M. J. Newby, A. C. Brombacher, and L. C. Tang, "Bivariate constant stress degradation model: led lighting system reliability estimation with two-stage modelling," Quality and Reliability Engineering International, vol. 25, no. 8, pp. 10671084, 2009.

[17] Z. Pan, N. Balakrishnan, and Q. Sun, "Bivariate constant-stress accelerated degradation model and inference," Communications in Statistics: Simulation and Computation, vol. 40, no. 2, pp. 259269, 2011.

[18] Z. Pan, N. Balakrishnan, Q. Sun, and J. Zhou, "Bivariate degradation analysis of products based on Wiener processes and copulas," Journal of Statistical Computation and Simulation, vol. 83, no. 7, pp. 1316-1329, 2013.

[19] M. Crowder and J. Lawless, "On a scheme for predictive maintenance," European Journal of Operational Research, vol. 176, no. 3, pp. 1713-1722, 2007.

[20] J. M. van Noortwijk, "A survey of the application of gamma processes in maintenance," Reliability Engineering and System Safety, vol. 94, no. 1, pp. 2-21, 2009.

[21] C.-C. Tsai, S.-T. Tseng, and N. Balakrishnan, "Mis-specification analyses of gamma and Wiener degradation processes," Journal of Statistical Planning and Inference, vol. 141, no. 12, pp. 37253735, 2011.

[22] V. Bagdonavicius and M. S. Nikulin, "Estimation in degradation models with explanatory variables," Lifetime Data Analysis, vol. 7, no. 1, pp. 85-103, 2001.

[23] X. Wang, N. Balakrishnan, B. Guo, and P. Jiang, "Residual life estimation based on bivariate non-stationary gamma degradation process," Journal of Statistical Computation and Simulation, vol. 85, no. 2, pp. 405-421, 2015.

[24] R. B. Nelsen, An Introduction to Copulas, Springer, New York, NY, USA, 2006.

[25] G. A. Whitmore and F. Schenkelberg, "Modeling accelerated degradation data using Wiener diffusion with a time scale transformation," Lifetime Data Analysis, vol. 3, no. 1, pp. 27-45, 1997.

[26] W. K. Hastings, "Monte carlo sampling methods using Markov chains and their applications," Biometrika, vol. 57, no. 1, pp. 97109, 1970.

[27] A. F. Smith and G. O. Roberts, "Bayesian computation via the Gibbs sampler and related Markov chain Monte Carlo methods," Journal of the Royal Statistical Society. Series B, vol. 55, no. 1, pp. 3-23, 1993.

[28] I. Ntzoufras, Bayesian Modeling Using WinBUGS, John Wiley \& Sons, New York, NY, USA, 2009.

[29] V. N. H. Chaluvadi, Accelerated life testing of electronic revenue meters [Ph.D. thesis], Clemson University, Clemson, SC, USA, 2008.

[30] S. T. Tseng, J. Tang, and L. H. Ku, "Determination of optimal burn-in parameters and residual life for highly reliable products," Naval Research Logistics, vol. 50, pp. 1-14, 2003. 

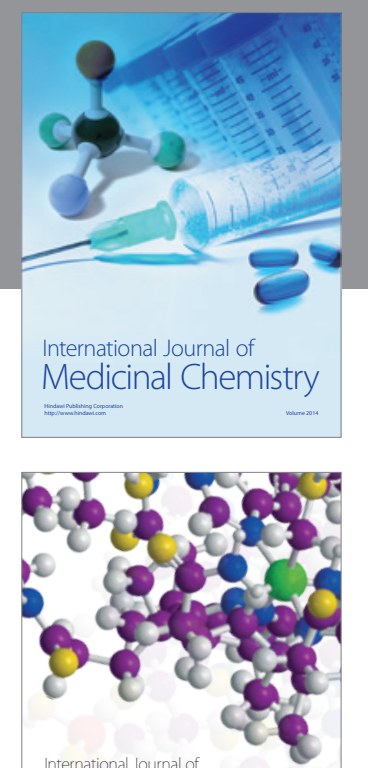

\section{Carbohydrate} Chemistry

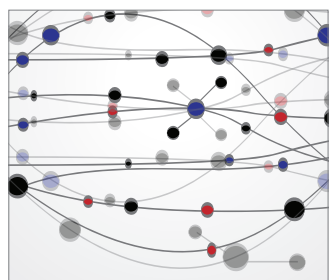

The Scientific World Journal
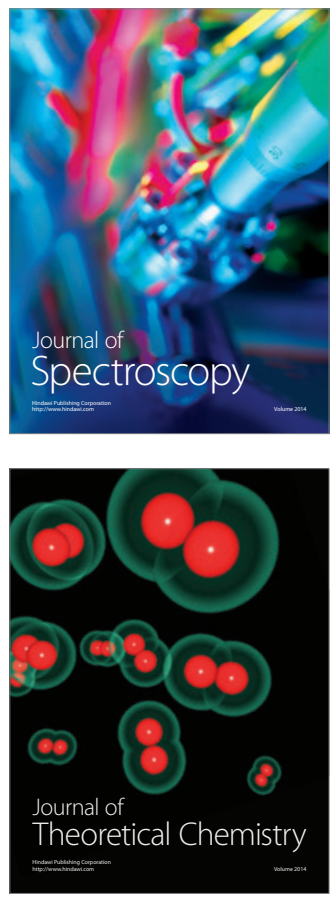
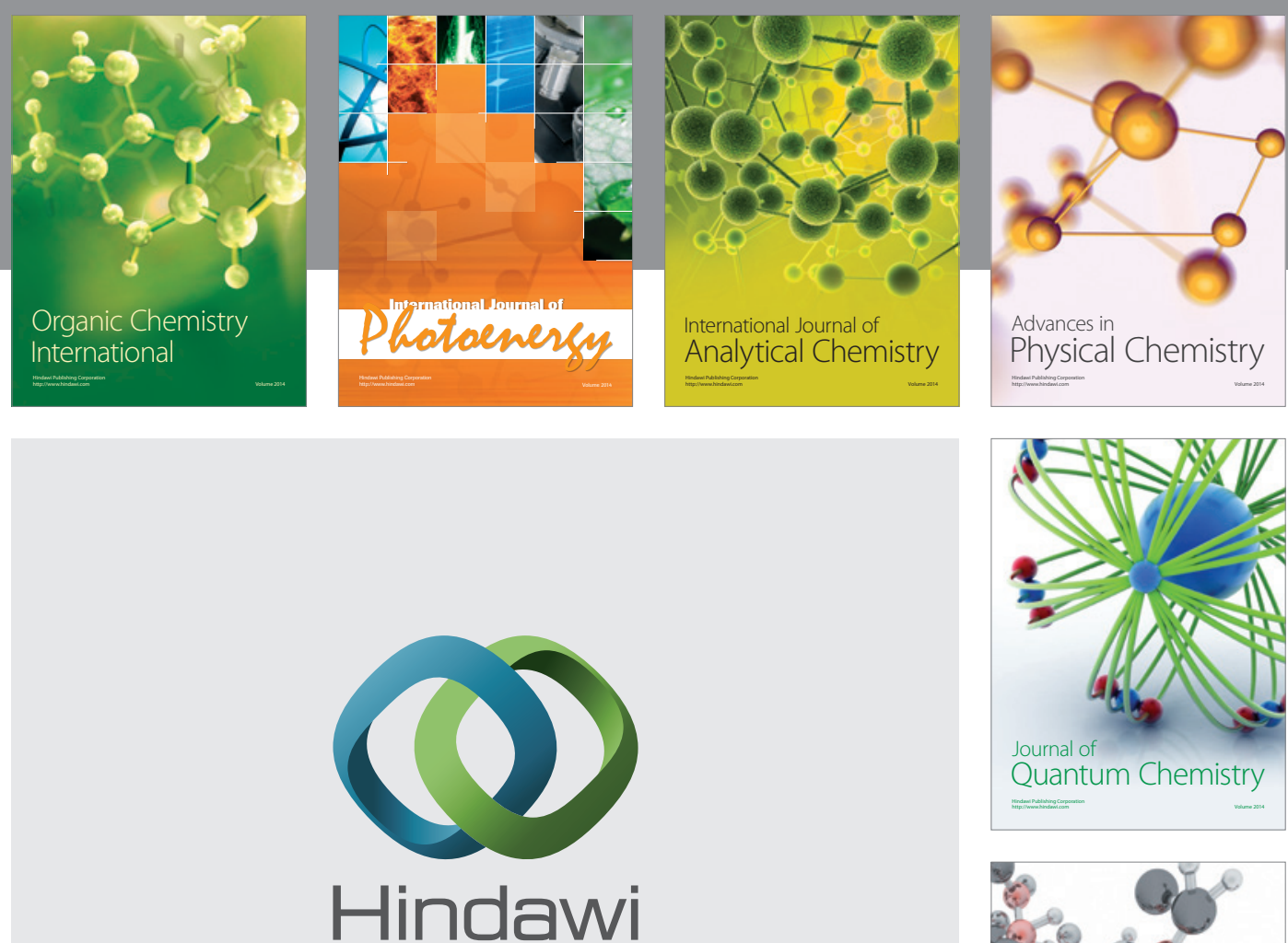

Submit your manuscripts at

http://www.hindawi.com

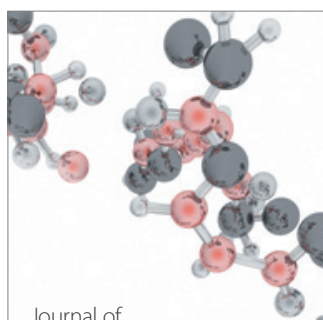

Analytical Methods

in Chemistry

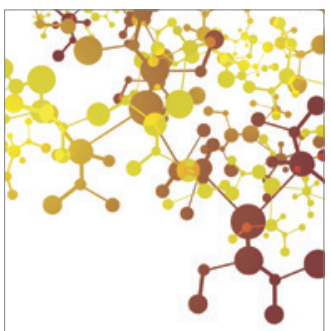

Journal of

Applied Chemistry

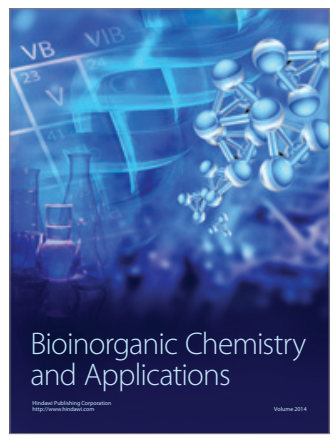

Inorganic Chemistry
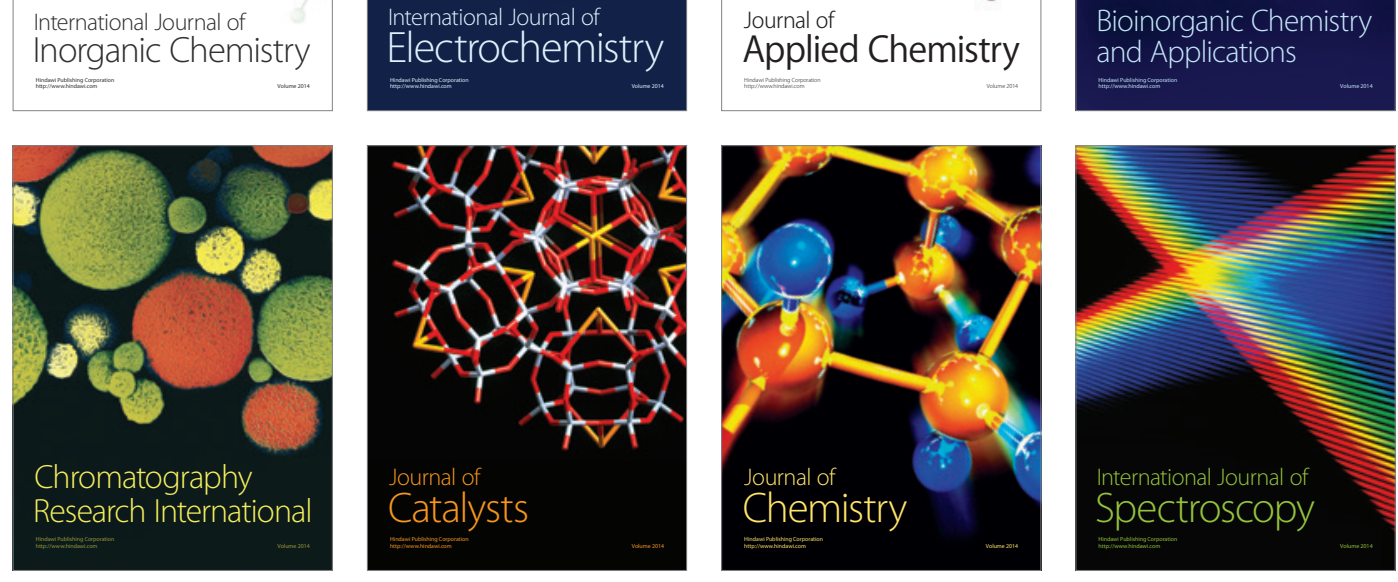